\title{
Mosapride Improves Lower Esophageal Sphincter and Esophageal Body Function in Patients With Minor Disorders of Esophageal Peristalsis
}

\author{
Sung Eun Kim, ${ }^{1,2}$ Moo In Park, ${ }^{1,2 *}$ Seun Ja Park, ${ }^{1,2}$ Won Moon, ${ }^{1,2}$ Jae Hyun Kim, ${ }^{1,2}$ Kyoungwon Jung, ${ }^{1,2}$ Hye Jung Kwon, ${ }^{2}$ \\ Gyung Mi Kim, ${ }^{2}$ and Hee Kyoung Joo ${ }^{2}$ \\ ${ }^{I}$ Department of Internal Medicine, Kosin University College of Medicine, Busan, Korea; and ${ }^{2}$ Department of Internal Medicine, Kosin University \\ Gospel Hospital, Busan, Korea
}

\begin{abstract}
Background/Aims
High-resolution manometry (HRM) has broadened the awareness of minor esophageal peristaltic disorders. However, the treatments for these minor disorders are limited and the role of prokinetics has been controversial. This study evaluates the effect of mosapride in patients with minor peristaltic disorders.
\end{abstract}

\section{Methods}

This study prospectively enrolled 21 patients with esophageal symptoms who were diagnosed with minor peristaltic disorders by gastroscopy and HRM using the Chicago classification version 3.0. Patients received mosapride $30 \mathrm{mg}$ daily for 2 weeks. Symptoms were assessed using the abbreviated World Health Organization quality of life scale (WHOQOL-BREF) and a HRM study was performed before and after 2 weeks of treatment.

\section{Results}

HRM metrics of lower esophageal sphincter (LES) respiratory mean pressure (median $14.6 \mathrm{mmHg}$ vs $17.3 \mathrm{mmHg}$; interquartile range [IQR] $8.7-22.5 \mathrm{mmHg}$ vs $12.5-25.9 \mathrm{mmHg} ; P=0.004$ ) and distal contractile integral (median $343.8 \mathrm{mmHg} \cdot \mathrm{sec} \cdot \mathrm{cm}$ vs 698.1 $\mathrm{mmHg} \cdot \mathrm{sec} \cdot \mathrm{cm} ;$ IQR $286.5-795.9 \mathrm{mmHg} \cdot \mathrm{sec} \cdot \mathrm{cm}$ vs $361.0-1127.6 \mathrm{mmHg} \cdot \mathrm{sec} \cdot \mathrm{cm} ; P=0.048$ ) were significantly increased after treatment. Complete response $(\geq 80.0 \%)$, satisfactory response $(\geq 50.0 \%)$, partial response $(<50.0 \%)$, and refractory response rates were $19.0 \%, 52.4 \%, 14.3 \%$, and $14.3 \%$, respectively. However, there was no statistical difference in all WHOQOL-BREF scores before and after treatment. Univariate analysis showed LES respiratory mean pressure $(P=0.036)$ was associated with symptom improvement (complete + satisfactory group). However, no statistical difference was found in other factors after multivariate analysis.

\section{Conclusions}

Mosapride improved esophageal symptoms and significantly increased LES respiratory mean pressure and distal contractile integral. Therefore, mosapride could enhance LES and esophageal body contraction pressures in patients with minor peristaltic disorders.

(J Neurogastroenterol Motil 2020;26:232-240)

Key Words

Esophageal motility disorders; Esophageal sphincter, lower; Manometry; Mosapride; Symptom assessment

Received: March 21, 2019 Revised: July 2, 2019 Accepted: September 20, 2019

(a) This is an Open Access article distributed under the terms of the Creative Commons Attribution Non-Commercial License (http:// creativecommons. org/licenses/by-nc/4.0) which permits unrestricted non-commercial use, distribution, and reproduction in any medium, provided the original work is properly cited.

${ }^{*}$ Correspondence: Moo In Park, MD, PhD

Department of Internal Medicine, Kosin University College of Medicine, 262 Gamcheon-ro, Seo-gu, Busan 49267, Korea Tel: +82-51-990-6719, Fax: +82-51-990-5055, E-mail: mipark@ns.kosinmed.or.kr 


\section{Introduction}

Esophageal high-resolution manometry (HRM) has been used to express pressure phenomena, bolus transit, and peristalsis in the esophagus with color pressure topography plots. ${ }^{1}$ With advances in HRM, researchers have gained an understanding of esophageal motility disorders. In 2009, the HRM Working Group suggested the Chicago classification (CC) to investigate and categorize esophageal motility disorders using key esophageal pressure topography metrics. The $\mathrm{CC}$ has been continuously upgraded through $2014 .^{2-4}$

Compared to the previous version, one of the changes in the CC version 3.0 (v3.0) simplified the criteria for the diagnosis of minor disorders of peristalsis. Peristaltic abnormalities were classified into 5 categories in the previous version. ${ }^{3}$ However, minor peristaltic disorders include ineffective esophageal motility (IEM) and fragmented peristalsis in CC v3.0, both of which present as poor bolus transit in the esophagus. ${ }^{4}$

The clinical interpretation of minor peristaltic disorders remains unclear. ${ }^{5}$ Numerous studies have been conducted on achalasia and major disorders of peristalsis, such as absent contractility and distal esophageal spasm. In contrast, few studies have examined minor disorders of peristalsis. Moreover, the management of minor disorders of peristalsis has been challenging. Minor disorders of peristalsis are frequently accompanied by gastroesophageal reflux due to delayed esophageal clearance. Therefore, proton pump inhibitors (PPIs) and potent antisecretory agents, have been used to control symptoms and associated complications in patients with minor peristaltic disorders. ${ }^{6}$ Several studies have evaluated the effectiveness of a few prokinetic agents on esophageal motility in healthy volunteers and patients with gastroesophageal reflux disease (GERD) ${ }^{6-8}$ However, few studies have investigated the usefulness of prokinetics in minor disorders of peristalsis.

Mosapride, a prokinetic agent, is known to enhance lower esophageal sphincter (LES) resting pressure and provoke esophageal body contractions. ${ }^{9}$ Unfortunately, mosapride revealed different results in esophageal motility in healthy volunteer studies. ${ }^{10,11}$ There have been a few studies using mosapride to investigate GERD patients, however, few studies have evaluated patients with minor disorders of peristalsis using mosapride. . $^{12,13}$

The aims of this study are to determine the efficacy of mosapride in patients with minor peristaltic disorders and to verify the factors associated with symptom improvement.

\section{Materials and Methods}

\section{Study Design and Subjects}

Between April 2016 and November 2017, this study prospectively enrolled patients aged $\geq 18$ years who underwent upper endoscopy with esophageal HRM and were diagnosed with minor disorders of peristalsis according to the $\mathrm{CC}$ v 3.0 at our hospital. All of the patients had typical or atypical symptoms of GERD and/or dysphagia. Typical reflux symptoms included acid regurgitation and heartburn, and atypical reflux symptoms included throat discomfort and cough. Exclusion criteria were (1) age $<18$ years; (2) severe systemic disease, except for hypertension and diabetes mellitus, requiring uninterrupted medication; (3) previous gastrointestinal malignancy; (4) previous upper gastrointestinal surgery; (5) alarm symptoms, including weight loss or recurrent vomiting; (6) esophageal stricture; or (7) previous esophageal procedures, including balloon dilation and surgical or endoscopic myotomy of the LES.

Before the administration of mosapride, all patients completed a questionnaire under the supervision of a trained interviewer. The patients were then administered $10 \mathrm{mg}$ of oral mosapride 3 times per day for 2 weeks. All enrolled patients stopped acid suppression medications while administering mosapride. After 2 weeks, all patients repeated the questionnaire and the HRM study was performed again.

The study protocol was reviewed and approved by the Institutional Review Board (IRB) of the Kosin University Gospel Hospital (IRB No. 2014-07-093) and informed consent was obtained from all participants.

\section{Endoscopic Examination}

All participants underwent esophagogastroduodenoscopy (GIF H260; Olympus, Tokyo, Japan) before participating in this study. Erosive esophagitis was graded using the Los Angeles classification. Hiatal hernia was defined as a distance between the esophagogastric junction and the diaphragmatic impingement of more than 2 $\mathrm{cm} .{ }^{14}$ Endoscopy was performed by skilled practitioners.

\section{Questionnaire and Symptom Assessment}

The questionnaire collected data on smoking and alcohol use, bothersome symptoms (eg, dysphagia, chest discomfort, acid regurgitation, heartburn, globus, and hoarseness), and quality of life (QOL). The QOL was investigated using the translated and validated Korean version of the abbreviated World Health Organization quality 
of life scale (WHOQOL-BREF). ${ }^{15}$ The WHOQOL-BREF assesses domains of overall QOL, physical health, psychological health, social relationships, and environmental QOL. Each question is scored using a 5-point Likert scale. The scores are reported on a linear scale (range 0-100), and higher scores indicate better QOL.

Before and after 2 weeks of mosapride treatment, patients were asked to express changes in the most bothersome symptoms as a percentage. The response to mosapride treatment was categorized as either complete ( $>80.0 \%$ symptom improvement), satisfactory ( $>50.0 \%$ improvement), partial ( $<50.0 \%$ improvement), or refractory (unresponsive to mosapride). Patients with a complete or satisfactory response were defined as responders, and patients with partial or refractory response were defined as non-responders. ${ }^{15}$

\section{Esophageal High-resolution Manometry Protocol and Data Analysis}

Acid suppression medications, including PPIs and histamine-2 receptor antagonists were stopped at least 2 weeks prior to the HRM test. Esophageal HRM was performed by a trained nurse using a ManoScan 360 instrument (Sierra Scientific Instruments Inc, Los Angeles, CA, USA). Esophageal manometry was performed with a solid-state catheter and 36 circumferential pressure sensors at $1-\mathrm{cm}$ intervals. Participants fasted overnight before the HRM test and were examined in the sitting position. The catheter was calibrated and positioned from the pharynx to the stomach transnasally. Therefore, the most proximal $1-\mathrm{cm}$ interval pressure sensors were located $2 \mathrm{~cm}$ above the LES. After calibration, participants performed 10 swallows of a $5-\mathrm{mL}$ water bolus at ambient temperature, at intervals of 30 seconds.

The primary outcome was a demonstration of a change in esophageal motility or peristalsis using HRM and the secondary outcome was an improvement of symptoms and a change in QOL. ManoView analysis software (Sierra Scientific Instruments Inc) was used to analyze data. According to $\mathrm{CC}$ v3.0, IEM was defined as ineffective swallows $\geq 50.0 \%$, including failed or weak contraction vigor with distal contractile integral (DCI) $<450 \mathrm{mmHg} \cdot \mathrm{sec} \cdot \mathrm{cm}$. In addition, the fragmented peristalsis was defined as fragmented contractions $\geq 50.0 \%$, with DCI $>450 \mathrm{mmHg} \cdot \mathrm{sec} \cdot \mathrm{cm}$. Fragmented contraction was defined by large breaks (more than $5 \mathrm{~cm}$ in length) in the $20-\mathrm{mmHg}$ isobaric contour with DCI $>450$ mmHg.sec.cm. We used a mean baseline value of HRM metrics including LES respiratory mean pressure which was a mean pressure throughout the respiratory cycles. And, it was used to identify the factors predicting symptom improvement. All HRM data were reviewed by 2 experienced investigators (M.I.P. and S.E.K.).

\section{Sample Size}

Based on a previous esophageal manometry study, ${ }^{16}$ we determined that at least 16 participants would be necessary to identify a statistically significant difference with a power of $80.0 \%$. Therefore, a sample size of 22 participants was planned, assuming a $30.0 \%$ dropout rate.

\section{Statistical Methods}

Statistical analyses were conducted using the Statistical Package for the Social Sciences for Windows version 18.0 (IBM Corp, Armonk, NY, USA). Continuous parameters were presented as median (interquartile range $[\mathrm{IQR}]$ ) and analyzed with the Student's $t$ test or Mann-Whitney $U$ test. Categorical parameters were presented as number $(\%)$ and the chi-squared $\left(\chi^{2}\right)$ test or Fisher's exact test was used to compare the proportion of categorical parameters. The Wilcoxon signed-rank test was used to analyze statistical comparisons between baseline and after mosapride treatment. Univariate and multivariate logistic regression analyses were performed to determine predictive factors for symptom improvement after mosapride administration, presented as adjusted odds ratio (OR) and $95 \%$ confidence interval $(\mathrm{CI})$, with $P<0.05$ considered statistically significant.

\section{Results}

\section{Effect of Mosapride on Esophageal Lower Esophageal Sphincter Pressure, Distal Contraction, and Quality of Life}

This study enrolled 21 patients with minor peristaltic disorders who were administered mosapride. Of these, 15 had IEM and 6 had fragmented peristalsis. There were no adverse events from the administration of mosapride.

Baseline characteristics of 21 patients (13 males; median age $[\mathrm{IQR}]=55.0[44.5-60.0]$ years) are shown in Table 1. There were no significant differences in baseline demographic variables between the IEM and fragmented peristalsis groups (Table 1).

Table 2 shows the effect of mosapride, verified using HRM. The median LES respiratory pressure at baseline was $14.6 \mathrm{mmHg}$ and significantly increased to $17.3 \mathrm{mmHg}$ after mosapride administration ( $P=0.004$; Fig. 1$)$. In addition, the median DCI at baseline was $343.8 \mathrm{mmHg} \cdot \mathrm{sec} \cdot \mathrm{cm}$ and significantly increased to $698.1 \mathrm{mmHg} \cdot \mathrm{sec} \cdot \mathrm{cm}$ after mosapride administration $(P=0.048$; Fig. 2). However, there was no significant increase in other HRM 
Table 1. Baseline Characteristics in Patients With Minor Disorders of Peristalsis

\begin{tabular}{|c|c|c|c|c|}
\hline Characteristics & Total $(\mathrm{N}=21)$ & $\operatorname{IEM}(\mathrm{n}=15)$ & Fragmented peristalsis $(n=6)$ & $P$-value \\
\hline Age (yr) & $55.0(44.5-60.0)$ & $54.0(40.0-60.0)$ & $56.0(42.3-62.5)$ & 0.910 \\
\hline Gender & & & & 0.410 \\
\hline Male & $13(61.9)$ & $10(66.7)$ & $3(50.0)$ & \\
\hline Female & $8(38.1)$ & $5(33.3)$ & $3(50.0)$ & \\
\hline $\operatorname{BMI}\left(\mathrm{kg} / \mathrm{m}^{2}\right)$ & $22.6(21.1-26.9)$ & $22.4(20.8-29.3)$ & $23.1(21.1-25.1)$ & 0.970 \\
\hline Smoking & & & & 0.461 \\
\hline Current & $3(14.3)$ & $2(13.3)$ & $1(16.7)$ & \\
\hline Previous & $5(23.8)$ & $5(33.3)$ & $0(0.0)$ & \\
\hline Never & $13(61.9)$ & $8(53.3)$ & $5(83.3)$ & \\
\hline Alcohol & & & & 0.069 \\
\hline Current & $11(52.4)$ & $9(60.0)$ & $2(33.3)$ & \\
\hline Previous & $6(28.6)$ & $5(33.3)$ & $1(16.7)$ & \\
\hline Never & $4(19.0)$ & $1(6.7)$ & $3(50.0)$ & \\
\hline Most bothersome symptom & & & & 0.800 \\
\hline Dysphagia & $7(33.3)$ & $5(33.3)$ & $2(33.3)$ & \\
\hline Chest discomfort & $5(23.8)$ & $4(26.7)$ & $1(16.7)$ & \\
\hline Typical symptoms ${ }^{\mathrm{a}}$ & $6(28.6)$ & $4(26.7)$ & $2(33.3)$ & \\
\hline Atypical symptoms ${ }^{\mathrm{b}}$ & $3(14.3)$ & $2(13.3)$ & $1(16.7)$ & \\
\hline \multicolumn{5}{|l|}{ Endoscopic findings } \\
\hline Reflux esophagitis & & & & 0.658 \\
\hline Normal & $18(85.7)$ & $13(86.7)$ & $5(83.3)$ & \\
\hline LA grade A & $3(14.3)$ & $2(13.3)$ & $1(16.7)$ & \\
\hline Hiatal hernia & $3(14.3)$ & $1(6.7)$ & $2(33.3)$ & 0.184 \\
\hline
\end{tabular}

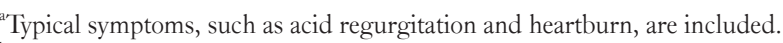

bAtypical symptoms, such as throat discomfort and cough, are included.

IEM, ineffective esophageal motility; BMI, body mass index; LA, Los Angeles.

Variables are presented as median (interquartile range) or number (\%).

variables including esophageal length, LES length, LES residual pressure, effective swallows, and intrabolus pressure $(P>0.05)$.

When IEM group and fragmented peristalsis group were analyzed separately, only the median LES respiratory pressure at baseline was significantly increased after mosapride administration $(14.3 \mathrm{mmHg}$ to $19.5 \mathrm{mmHg}, P=0.011$ ).

There was no significant increase in WHOQOL-BREF scores between baseline and after mosapride administration (Table $3)$. However, the overall score showed an increasing tendency $(P=$ $0.057)$.

\section{Mosapride Response Rates}

Table 4 shows the symptom response to mosapride in patients with minor peristaltic disorders. Four patients (19.0\%) had a complete response, $11(52.4 \%)$ had a satisfactory response, $3(14.3 \%)$ had a partial response, and $3(14.3 \%)$ had a refractory response. Accordingly, the proportions of responders to mosapride (71.4\%) were greater than the proportions of non-responders (28.6\%). However, no significant difference was revealed in the response rates between the IEM and fragmented peristalsis groups $(P=0.424)$.

\section{Factors Related to Symptom Improvement After Administration of Mosapride}

Of the 21 participants, 15 were responders and 6 were nonresponders. In univariate analysis, LES respiratory mean pressure $(P=0.036)$ was statistically correlated with symptom improvement (Table 5). However, no other factors were associated with symptom improvement. In addition, there were no significant associated factors in multivariate analysis (Table 5).

\section{Discussion}

The present study demonstrated that LES respiratory mean pressure and DCI were increased after mosapride treatment. In addition, the overall response rate (complete and satisfactory responses) to mosapride was $71.4 \%$. Although no factor predictive of 
Table 2. Effect of Mosapride on High-resolution Manometry Variables

\begin{tabular}{|c|c|c|c|c|c|c|c|c|c|}
\hline \multirow[b]{2}{*}{ HRM variables } & \multicolumn{3}{|c|}{ Total $(\mathrm{N}=21)$} & \multicolumn{3}{|c|}{$\operatorname{IEM}(\mathrm{n}=15)$} & \multicolumn{3}{|c|}{ Fragmented peristalsis $(n=6)$} \\
\hline & Baseline & $\begin{array}{c}\text { After } \\
\text { medication }\end{array}$ & $P$-value & Baseline & $\begin{array}{c}\text { After } \\
\text { medication }\end{array}$ & $P$-value & Baseline & $\begin{array}{c}\text { After } \\
\text { medication }\end{array}$ & $P$-value \\
\hline $\begin{array}{l}\text { Esophageal } \\
\text { length }(\mathrm{cm})\end{array}$ & $\begin{array}{c}27.8 \\
(27.0-29.0)\end{array}$ & $\begin{array}{c}28.0 \\
(26.6-29.1)\end{array}$ & 0.714 & $\begin{array}{c}28.1 \\
(27.1-29.3)\end{array}$ & $\begin{array}{c}28.6 \\
(27.1-29.2)\end{array}$ & 0.987 & $\begin{array}{c}27.1 \\
(25.2-27.4)\end{array}$ & $\begin{array}{c}26.5 \\
(24.6-27.2)\end{array}$ & 0.539 \\
\hline $\begin{array}{l}\text { LES length } \\
(\mathrm{cm})\end{array}$ & $\begin{array}{c}2.8 \\
(2.7-3.3)\end{array}$ & $\begin{array}{c}3.0 \\
(2.7-3.6)\end{array}$ & 0.130 & $\begin{array}{c}2.8 \\
(2.7-3.4)\end{array}$ & $\begin{array}{c}3.0 \\
(2.7-4.0)\end{array}$ & 0.241 & $\begin{array}{c}2.8 \\
(2.3-3.3)\end{array}$ & $\begin{array}{c}2.9 \\
(2.7-3.1)\end{array}$ & 0.779 \\
\hline $\begin{array}{l}\text { LES respiratory } \\
\text { mean pressure } \\
(\mathrm{mmHg})\end{array}$ & $\begin{array}{c}14.6 \\
(8.7-22.5)\end{array}$ & $\begin{array}{c}17.3 \\
(12.5-25.9)\end{array}$ & 0.004 & $\begin{array}{c}14.3 \\
(8.6-23.9)\end{array}$ & $\begin{array}{c}19.5 \\
(13.0-25.3)\end{array}$ & 0.011 & $\begin{array}{c}15.9 \\
(7.9-25.8)\end{array}$ & $\begin{array}{c}17.3 \\
(11.2-28.5)\end{array}$ & 0.436 \\
\hline $\begin{array}{l}\text { LES residual } \\
\text { pressure } \\
(\mathrm{mmHg})\end{array}$ & $\begin{array}{c}3.5 \\
(2.2-7.9)\end{array}$ & $\begin{array}{c}5.5 \\
(2.3-10.2)\end{array}$ & 0.192 & $\begin{array}{c}3.5 \\
(2.3-7.2)\end{array}$ & $\begin{array}{c}5.6 \\
(2.7-10.5)\end{array}$ & 0.197 & $\begin{array}{c}5.2 \\
(0.6-10.9)\end{array}$ & $\begin{array}{c}4.6 \\
(0.9-10.1)\end{array}$ & 0.411 \\
\hline $\begin{array}{l}\text { Effective } \\
\text { swallows (\%) }\end{array}$ & $\begin{array}{c}50.0 \\
(35.0-75.0)\end{array}$ & $\begin{array}{c}60.0 \\
(25.0-85.0)\end{array}$ & 0.321 & $\begin{array}{c}50.0 \\
(40.0-80.0)\end{array}$ & $\begin{array}{c}50.0 \\
(30.0-90.0)\end{array}$ & 0.757 & $\begin{array}{c}40.0 \\
(15.0-57.5)\end{array}$ & $\begin{array}{c}60.0 \\
(20.0-85.0)\end{array}$ & 0.274 \\
\hline $\begin{array}{l}\text { DCI } \\
(\mathrm{mmHg} \cdot \\
\mathrm{sec} \cdot \mathrm{cm})\end{array}$ & $\begin{array}{c}343.8 \\
(286.5-795.9)\end{array}$ & $\begin{array}{c}698.1 \\
(361.0-1127.6)\end{array}$ & 0.048 & $\begin{array}{c}334.7 \\
(285.4-460.2)\end{array}$ & $\begin{array}{c}785.5 \\
(405.7-1094.6)\end{array}$ & 0.137 & $\begin{array}{c}472.7 \\
(191.0-1535.8)\end{array}$ & $\begin{array}{c}675.7 \\
(297.5-1875.7)\end{array}$ & 0.088 \\
\hline $\begin{array}{l}\text { Intrabolus } \\
\text { pressure } \\
(\mathrm{mmHg})\end{array}$ & $\begin{array}{c}-0.7 \\
(-2.3-2.1)\end{array}$ & $\begin{array}{c}-1.4 \\
(-3.0-1.7)\end{array}$ & 0.320 & $\begin{array}{c}-0.6 \\
(-2.2-2.7)\end{array}$ & $\begin{array}{c}-1.4 \\
(-2.2-1.9)\end{array}$ & 0.390 & $\begin{array}{c}-0.4 \\
(-3.6-0.7)\end{array}$ & $\begin{array}{c}-1.2 \\
(-5.0-0.5)\end{array}$ & 0.489 \\
\hline
\end{tabular}

HRM, high-resolution manometry; IEM, ineffective esophageal motility; LES, lower esophageal sphincter; DCI, distal contractile integral.

Variables are presented as median (interquartile range).

Variables were compared using the non-parametric Wilcoxon signed rank test and a $P$-value $<0.05$ was considered significant.

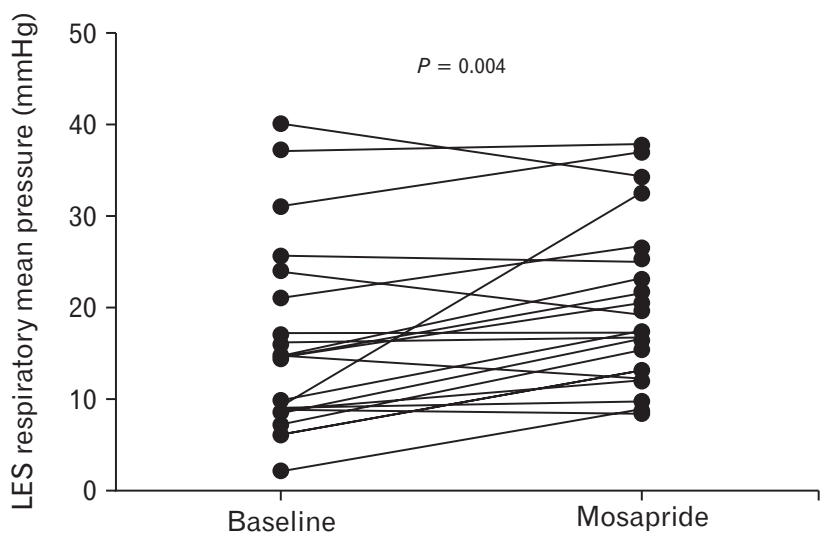

Figure 1. Median lower esophageal sphincter (LES) respiratory mean pressure $(\mathrm{mmHg})$ before and after mosapride administration.

symptom improvement after mosapride administration was identified using multivariate analysis, LES respiratory mean pressure was associated with improvement in univariate analysis. It is difficult to compare these results directly with those of previous studies. However, similar results were obtained in some studies that examined the usefulness of prokinetics in minor peristaltic disorders. To the best of our knowledge, no other study has analyzed the factors predictive

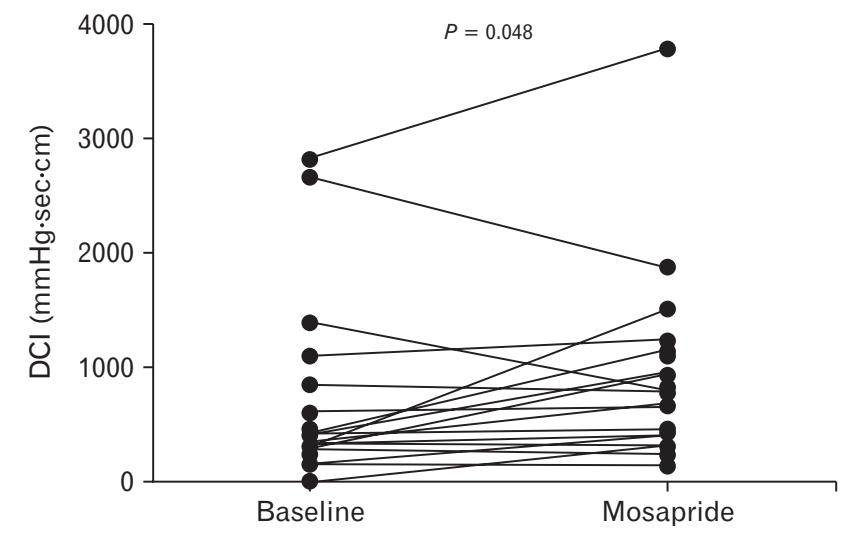

Figure 2. Median distal contractile integral (DCI, $\mathrm{mmHg} \cdot \mathrm{sec} \cdot \mathrm{cm})$ before and after mosapride administration.

of symptom improvement after mosapride treatment. Prokinetics have been used to treat minor peristaltic disorders. Ineffective peristalsis, based on a standard distal esophageal amplitude (DEA) criterion of $30 \mathrm{mmHg}$, was shown to have $85.0 \%$ sensitivity and $64.0 \%$ specificity, and $85.0 \%$ sensitivity and $66.0 \%$ specificity, for complete bolus transit during liquid swallows and viscous swallows, respectively. ${ }^{17}$ Therefore, increased DEA reflects complete bolus 
Table 3. Effect of Mosapride on Quality of Life

\begin{tabular}{|c|c|c|c|c|c|c|c|c|c|}
\hline \multirow{2}{*}{$\begin{array}{l}\text { WHOQOL-BREF } \\
\text { score }\end{array}$} & \multicolumn{3}{|c|}{ Total $(\mathrm{n}=21)$} & \multicolumn{3}{|c|}{$\operatorname{IEM}(\mathrm{n}=15)$} & \multicolumn{3}{|c|}{ Fragmented peristalsis $(\mathrm{n}=6)$} \\
\hline & Baseline & After medication & $P$-value & Baseline & After medication & $P$-value & Baseline & After medication & $P$-value \\
\hline Total & $\begin{array}{c}80.0 \\
(71.5-92.0)\end{array}$ & $\begin{array}{c}83.0 \\
(68.5-93.0)\end{array}$ & 0.631 & $\begin{array}{c}80.0 \\
(72.0-95.0)\end{array}$ & $\begin{array}{c}83.0 \\
(74.0-95.0)\end{array}$ & 0.401 & $\begin{array}{c}80.0 \\
(68.8-90.8)\end{array}$ & $\begin{array}{c}77.5 \\
(67.5-89.3)\end{array}$ & 0.621 \\
\hline Overall quality of life & $\begin{array}{c}6.0 \\
(5.0-7.0)\end{array}$ & $\begin{array}{c}6.0 \\
(6.0-7.0)\end{array}$ & 0.057 & $\begin{array}{c}5.0 \\
(5.0-7.0)\end{array}$ & $\begin{array}{c}6.0 \\
(6.0-7.0)\end{array}$ & 0.233 & $\begin{array}{c}6.0 \\
(7.8-6.3)\end{array}$ & $\begin{array}{c}6.5 \\
(6.0-7.3)\end{array}$ & 0.111 \\
\hline Physical health & $\begin{array}{c}22.0 \\
(18.0-24.0)\end{array}$ & $\begin{array}{c}22.0 \\
(17.0-24.5)\end{array}$ & 0.724 & $\begin{array}{c}22.0 \\
(19.0-24.0)\end{array}$ & $\begin{array}{c}23.0 \\
(18.0-25.0)\end{array}$ & 0.735 & $\begin{array}{c}21.0 \\
(16.8-24.0)\end{array}$ & $\begin{array}{c}19.0 \\
(17.0-23.3)\end{array}$ & 0.465 \\
\hline Psychological health & $\begin{array}{c}18.0 \\
(15.5-20.5)\end{array}$ & $\begin{array}{c}18.0 \\
(14.5-20.0)\end{array}$ & 0.333 & $\begin{array}{c}18.0 \\
(15.0-21.0)\end{array}$ & $\begin{array}{c}18.0 \\
(13.0-20.0)\end{array}$ & 0.178 & $\begin{array}{c}17.5 \\
(15.0-19.8)\end{array}$ & $\begin{array}{c}17.0 \\
(14.8-20.5)\end{array}$ & 0.741 \\
\hline Social relationships & $\begin{array}{c}11.0 \\
(9.0-12.0)\end{array}$ & $\begin{array}{c}11.0 \\
(9.0-12.0)\end{array}$ & 0.512 & $\begin{array}{c}11.0 \\
(9.0-12.0)\end{array}$ & $\begin{array}{c}11.0 \\
(9.0-12.0)\end{array}$ & 1.000 & $\begin{array}{c}10.5 \\
(9.0-12.5)\end{array}$ & $\begin{array}{c}10.0 \\
(9.0-11.5)\end{array}$ & 0.296 \\
\hline $\begin{array}{l}\text { Environmental } \\
\text { quality of life }\end{array}$ & $\begin{array}{c}26.0 \\
(21.5-29.0)\end{array}$ & $\begin{array}{c}26.0 \\
(21.0-30.0)\end{array}$ & 0.370 & $\begin{array}{c}26.0 \\
(22.0-29.0)\end{array}$ & $\begin{array}{c}26.0 \\
(23.0-30.0)\end{array}$ & 0.095 & $\begin{array}{c}26.0 \\
(20.5-29.0)\end{array}$ & $\begin{array}{c}23.0 \\
(20.0-28.0)\end{array}$ & 0.391 \\
\hline
\end{tabular}

WHOQOL-BREF, World Health Organization quality of life scale abbreviated version; IEM, ineffective esophageal motility.

Variables are presented as median (interquartile range).

Variables were compared using the non-parametric Wilcoxon signed rank test and a $P$-value $<0.05$ was considered significant.

Table 4. Symptom Responses to Mosapride According to the Subtype of Minor Disorders of Peristalsis

\begin{tabular}{ccccr}
\hline $\begin{array}{c}\text { Mosapride } \\
\text { response }\end{array}$ & $\begin{array}{c}\text { IEM } \\
(\mathrm{n}=15)\end{array}$ & $\begin{array}{c}\text { Fragmented } \\
\text { peristalsis } \\
(\mathrm{n}=6)\end{array}$ & $P$-value & $\begin{array}{c}\text { Overall } \\
\text { response } \\
\text { rate }\end{array}$ \\
\hline Responders & $10(66.7)$ & $5(83.4)$ & 0.424 & $15(71.4)$ \\
Complete & $3(20.0)$ & $1(16.7)$ & & $4(19.0)$ \\
Satisfactory & $7(46.7)$ & $4(66.7)$ & $11(52.4)$ \\
Non-responders & $5(43.3)$ & $1(16.7)$ & $6(28.6)$ \\
Partial & $3(20.0)$ & $0(0.0)$ & $3(14.3)$ \\
Refractory & $2(13.3)$ & $1(16.7)$ & $3(14.3)$ \\
\hline
\end{tabular}

IEM, ineffective esophageal motility.

Variables are presented as number $(\%)$.

transit with high specificity. Results of the present study correspond with the results of earlier studies reporting that prokinetics can be useful as a treatment for minor disorders of peristalsis. Agrawal et $\mathrm{al}^{18}$ showed that bethanechol, a direct-acting muscarinic receptor agonist, significantly improved bolus transit in patients with severe IEM by increasing DEA. Although presently unavailable, cisapride, a 5-hydroxytryptamine $4\left(5-\mathrm{HT}_{4}\right)$ receptor agonist, significantly enhanced ineffective esophageal peristalsis along with bolus clearance and DEA in patients with nonspecific esophageal motility disorders. ${ }^{19}$ A recent study also revealed that another $5-\mathrm{HT}_{4}$ receptor agonist, prucalopride, significantly increased pressure wave amplitude and the peristaltic success rate in patients with IEM. ${ }^{20} \mathrm{~A}$ double-blind, randomized study in healthy volunteers reported that buspirone (5- $\mathrm{HT}_{1 \mathrm{~A}}$ and $5-\mathrm{HT}_{2}$ receptor agonists), bethanechol, and pyridostigmine (a cholinesterase inhibitor) significantly enhanced mean DEA and mean LES residual pressure. ${ }^{8}$ Therefore, it is presumed that prokinetic agents could be used to treat minor disorders of peristalsis by enhancing esophageal motility.

Mosapride enhances acetylcholine release at the gastrointestinal neuromuscular junction by promoting $5-\mathrm{HT}_{4}$ receptor activity, elevates LES resting pressure, and affects esophageal body contractions. ${ }^{9}$ The results in healthy volunteers were unclear. One study showed that mosapride increased esophageal motility and facilitated bolus transit in the esophagus, ${ }^{10}$ while another study reported that mosapride did not stimulate esophageal motility. ${ }^{11}$ In patients with chronic GERD, mosapride significantly increased the esophageal contraction amplitude and the contractile duration. ${ }^{9}$ Interestingly, a rodent study demonstrated that mosapride reduced mucosal inflammation in a rat model of reflux esophagitis by decreasing the number of endothelin-1-positive monocytes in the esophagus. ${ }^{21}$ Chen et al ${ }^{13,22}$ reported that mosapride enhanced esophageal sensitivity to distension-related secondary peristalsis in both healthy volunteers and patients with IEM. Although the effects of mosapride on secondary peristaltic contractions are limited in IEM patients, the pressure wave amplitude and rate of complete peristalsis were significantly increased with respect to primary peristalsis after mosapride treatment. ${ }^{13}$ It is inferred that mosapride, like other prokinetic agents, may also affect esophageal motility in patients with IEM.

An earlier study by our group reported a responder rate of $54.2 \%$ for minor peristaltic disorders in patients treated with anti- 
Table 5. Factors Predicting Symptom Improvement With Mosapride Treatment

\begin{tabular}{|c|c|c|c|c|c|}
\hline Variables & $\begin{array}{l}\text { Responders } \\
(\mathrm{n}=15)\end{array}$ & $\begin{array}{l}\text { Non-responders } \\
\quad(\mathrm{n}=6)\end{array}$ & $\begin{array}{l}\text { Univariated } \\
\text { analysis } \\
P \text {-value }\end{array}$ & $\begin{array}{c}\text { Multivariated } \\
\text { analysis } \\
P \text {-value }\end{array}$ & $\begin{array}{c}\text { Adjusted } \\
\text { OR }(95 \% \mathrm{CI})^{\mathrm{b}}\end{array}$ \\
\hline Age (yr) & $55.0(49.0-60.0)$ & $55.5(39.5-60.3)$ & 0.970 & 0.243 & $0.94(0.85-1.04)$ \\
\hline Female & $7(46.7)$ & $1(16.7)$ & 0.221 & 0.758 & $1.65(0.07-39.72)$ \\
\hline $\operatorname{BMI}\left(\mathrm{kg} / \mathrm{m}^{2}\right)$ & $22.9(21.5-26.7)$ & $22.2(20.2-27.8)$ & 0.850 & - & - \\
\hline Smoking (current + previous) & $5(33.3)$ & $3(50.0)$ & 0.410 & - & - \\
\hline Alcohol (current + previous) & $12(80.0)$ & $5(83.3)$ & 0.684 & - & - \\
\hline Reflux esophagitis, LA grade A & $2(13.3)$ & $1(16.7)$ & 0.658 & - & - \\
\hline Hiatal hernia & $2(13.3)$ & $1(16.7)$ & 0.658 & - & - \\
\hline Fragmented peristalsis & $5(33.3)$ & $1(16.7)$ & 0.424 & 0.220 & $14.38(0.20-1021.30)$ \\
\hline \multicolumn{6}{|l|}{ WHOQOL-BREF baseline score } \\
\hline Total & $85.0(72.0-95.0)$ & $79.0(65.3-83.3)$ & 0.302 & - & - \\
\hline Overall quality of life & $6.0(5.0-7.0)$ & $5.5(4.8-6.3)$ & 0.569 & - & - \\
\hline Physical health & $22.0(19.0-24.0)$ & $21.5(16.5-23.3)$ & 0.519 & - & - \\
\hline Psychological health & $19.0(16.0-21.0)$ & $16.5(12.8-19.0)$ & 0.178 & - & - \\
\hline Social relationships & $11.0(9.0-12.0)$ & $10.5(8.8-12.0)$ & 0.677 & - & - \\
\hline Environmental quality of life & $26.0(22.0-29.0)$ & $23.5(20.0-27.5)$ & 0.302 & - & - \\
\hline \multicolumn{6}{|l|}{ HRM metrics } \\
\hline Esophageal length (cm) & $27.1(26.9-28.1)$ & $28.8(27.0-30.3)$ & 0.132 & - & - \\
\hline LES length $(\mathrm{cm})$ & $2.8(2.7-3.2)$ & $2.8(2.3-3.4)$ & 0.677 & - & - \\
\hline LES respiratory mean pressure $(\mathrm{mmHg})$ & $16.0(8.9-25.7)$ & $8.7(8.0-11.0)$ & 0.036 & 0.112 & $1.18(0.96-1.46)$ \\
\hline LES residual pressure $(\mathrm{mmHg})$ & $5.3(1.5-9.3)$ & $3.2(2.2-4.0)$ & 0.381 & 0.892 & $1.05(0.49-2.26)$ \\
\hline Effective swallows (\%) & $50.0(30.0-80.0)$ & $50.0(30.0-75.0)$ & 0.910 & - & - \\
\hline DCI (mmHg·sec·cm) & $338.0(288.8-1177.0)$ & $375.8(115.9-440.5)$ & 0.444 & 0.376 & $1.00(0.99-1.01)$ \\
\hline Intrabolus pressure (mmHg) & $-0.7(-2.4-2.6)$ & $-0.8(-2.7-2.4)$ & 0.841 & - & - \\
\hline
\end{tabular}

${ }^{a}$ Variables were compared using the non-parametric Fisher's exact test or Mann-Whitney test and a $P$-value $<0.05$ was considered significant.

${ }^{\mathrm{b}}$ Logistic model including terms of age, sex, subtypes of minor peristaltic disorders, lower esophageal sphincter (LES) respiratory mean pressure, LES residual pressure, and distal contractile integral (DCI).

OR, odds ratio; CI, confidence interval; BMI, body mass index; LA, Los Angeles; WHOQOL-BREF, World Health Organization quality of life scale abbreviated version; HRM, high-resolution manometry.

Variables are presented as median (interquartile range) or number $(\%)$.

reflux therapy using PPIs or prokinetics. ${ }^{23}$ Since the study was performed retrospectively, the results may vary from those in the current study. In addition, the results of this study are in close agreement with those in previous studies in patients with GERD. Double-blind randomized studies from Korea and Japan showed that symptomatic response rates in a mosapride treatment group were $79.2 \%$ and $88.6 \%$, respectively. ${ }^{12,24}$ However, there was no significant difference in the symptomatic response rate in each study compared to that in a placebo group. ${ }^{12,24}$ Thus, mosapride may improve symptoms in patients with esophageal motility disorders, but further research is required to determine whether this is a placebo effect.

Our study showed that LES respiratory mean pressure was significantly higher in mosapride responders. Jain et $\mathrm{al}^{25}$ demonstrated that basal LES pressure, integrated relaxation pressure, and hiatus size were associated with abnormal DeMeester scores in GERD patients. Among these, hypotensive basal LES pressure (< $10 \mathrm{mmHg}$ ), which reflects decreased esophageal function, was the sole factor predictive of pathologic acid reflux, with a likelihood ratio of 2.2..$^{25}$ According to pathogenesis, esophageal hypomotility disorders consist of 3 stages: neuropathy, myopathy, and fibrosis. ${ }^{26}$ As the disease stages progress, esophageal motor function is further reduced. Therefore, it is postulated that patients with more preserved esophageal function would have a greater benefit from mosapride treatment. As the number of patients in the current study was small, this is difficult to prove. However, the mosapride responders group had higher rates of fragmented peristalsis.

There are several limitations in the current study. First, there was no control group. Second, the duration of mosapride administration might not be enough. It would have been more meaningful 
if the patients had a longer duration of mosapride administration. Third, the number of patients with minor peristaltic disorders was small and no associated factors were found in multivariate analysis. In addition, we did not perform esophageal transit scintigraphy or a timed barium esophagogram. Through this study, it was presumed that mosapride could affect esophageal bolus transit by increasing DCI. This assumption needs to be verified in the future. Nonetheless, this study is strengthened by the comprehensive evaluation using upper endoscopy, HRM, questionnaires, and the attempt to identify predictive factors.

In conclusion, mosapride influences esophageal peristalsis by enhancing basal LES respiratory mean pressure and esophageal contraction pressure in patients with minor peristaltic disorders. Further large-scale randomized studies are required to establish the efficacy of mosapride in these patients.

Acknowlegements: We would like to thank Daewoong Pharmaceuticals Co, Ltd for supporting the study. The pharmaceutical company had no role in study design, data collection and analysis, decision to publish, or preparation of the manuscript.

\section{Financial support: None.}

\section{Conflicts of interest: None.}

Author contributions: Sung Eun Kim collected patients, analyzed the data, drafted the manuscript, tables, and figures, and approved the final version to be published; Moo In Park designed and supervised the study, collected patients, revised the draft, and approved the final version; Seun Ja Park, Won Moon, Jae Hyun Kim, Kyoungwon Jung, and Hye Jung Kwon reviewed the manuscript, and offered advice of the manuscript; Gyung Mi Kim and Hee Kyoung Joo performed esophageal motility studies; and all authors read and approved the final manuscript.

\section{References}

1. Gyawali CP. High resolution manometry: the Ray Clouse legacy. Neurogastroenterol Motil 2012;24(suppl 1):2-4.

2. Pandolfino JE, Fox MR, Bredenoord AJ, Kahrilas PJ. High-resolution manometry in clinical practice: utilizing pressure topography to classify oesophageal motility abnormalities. Neurogastroenterol Motil 2009;21:796-806.

3. Bredenoord AJ, Fox M, Kahrilas PJ, et al. Chicago classification criteria of esophageal motility disorders defined in high resolution esophageal pressure topography. Neurogastroenterol Motil 2012;24(suppl 1):57-65.

4. Kahrilas PJ, Bredenoord AJ, Fox M, et al. The Chicago classifica- tion of esophageal motility disorders, v3.0. Neurogastroenterol Motil 2015;27:160-174.

5. Ravi K, Friesen L, Issaka R, Kahrilas PJ, Pandolfino JE. Long-term outcomes of patients with normal or minor motor function abnormalities detected by high-resolution esophageal manometry. Clin Gastroenterol Hepatol 2015;13:1416-1423.

6. Maradey-Romero C, Gabbard S, Fass R. Treatment of esophageal motility disorders based on the chicago classification. Curr Treat Options Gastroenterol 2014;12:441-455.

7. Staiano A, Clouse RE. The effects of cisapride on the topography of oesophageal peristalsis. Aliment Pharmacol Ther 1996;10:875-882.

8. Blonski W, Vela MF, Freeman J, Sharma N, Castell DO. The effect of oral buspirone, pyridostigmine, and bethanechol on esophageal function evaluated with combined multichannel esophageal impedance-manometry in healthy volunteers. J Clin Gastroenterol 2009;43:253-260.

9. Ruth M, Finizia C, Cange L, Lundell L. The effect of mosapride on oesophageal motor function and acid reflux in patients with gastrooesophageal reflux disease. Eur J Gastroenterol Hepatol 2003;15:11151121.

10. Cho YK, Choi MG, Han HW, et al. The effect of mosapride on esophageal motility and bolus transit in asymptomatic volunteers. J Clin Gastroenterol 2006;40:286-292.

11. Koshino K, Adachi K, Furuta K, et al. Effects of mosapride on esophageal functions and gastroesophageal reflux. J Gastroenterol Hepatol 2010;25:1066-1071.

12. Cho YK, Choi MG, Park EY, et al. Effect of mosapride combined with esomeprazole improves esophageal peristaltic function in patients with gastroesophageal reflux disease: a study using high resolution manometry. Dig Dis Sci 2013;58:1035-1041.

13. Chen CL, Yi CH, Liu TT, Orr WC. Effects of mosapride on secondary peristalsis in patients with ineffective esophageal motility. Scand J Gastroenterol 2013;48:1363-1370.

14. Hyun JJ, Bak YT. Clinical significance of hiatal hernia. Gut Liver 2011;5:267-277.

15. Kim SE, Kim N, Oh S, et al. Predictive factors of response to proton pump inhibitors in korean patients with gastroesophageal reflux disease. J Neurogastroenterol Motil 2015;21:69-77.

16. Fukazawa K, Furuta K, Adachi K, et al. Effects of mosapride on esophageal motor activity and esophagogastric junction compliance in healthy volunteers. J Gastroenterol 2014;49:1307-1313.

17. Tutuian R, Castell DO. Clarification of the esophageal function defect in patients with manometric ineffective esophageal motility: studies using combined impedance-manometry. Clin Gastroenterol Hepatol 2004;2:230-236.

18. Agrawal A, Hila A, Tutuian R, Mainie I, Castell DO. Bethanechol improves smooth muscle function in patients with severe ineffective esophageal motility. J Clin Gastroenterol 2007;41:366-370.

19. Song CW, Um SH, Kim CD, Ryu HS, Hyun JH, Choe JG. Doubleblind placebo-controlled study of cisapride in patients with nonspecific esophageal motility disorder accompanied by delayed esophageal transit. Scand J Gastroenterol 1997;32:541-546.

20. Lei WY, Hung JS, Liu TT, Yi CH, Chen CL. Influence of prucalo- 
pride on esophageal secondary peristalsis in reflux patients with ineffective motility. J Gastroenterol Hepatol 2018;33:650-655.

21. Zhuang ZH, Zou FM, Tang DP, Zhuang JY, Wei JJ, Yang LY. The $5-\mathrm{HT}_{4}$ receptor agonist mosapride attenuates inflammation of reflux esophagitis. Hepatogastroenterology 2014;61:115-119.

22. Chen CL, Liu TT, Yi CH, Orr WC. Effects of mosapride on esophageal secondary peristalsis in humans. Neurogastroenterol Motil 2011;23:606e249.

23. Jeong J, Kim SE, Park MI, et al. The effect of anti-reflux therapy on patients diagnosed with minor disorders of peristalsis in high-resolution manometry. Korean J Gastroenterol 2017;69:212-219.
24. Miwa H, Inoue K, Ashida K, et al. Randomised clinical trial: efficacy of the addition of a prokinetic, mosapride citrate, to omeprazole in the treatment of patients with non-erosive reflux disease - a double-blind, placebocontrolled study. Aliment Pharmacol Ther 2011;33:323-332.

25. Jain M, Srinivas M, Bawane P, Venkataraman J. Basal lower esophageal sphincter pressure in gastroesophageal reflux disease: an ignored metric in high-resolution esophageal manometry. Indian J Gastroenterol 2018;37:446-451.

26. Sallam H, McNearney TA, Chen JD. Systematic review: pathophysiology and management of gastrointestinal dysmotility in systemic sclerosis (scleroderma). Aliment Pharmacol Ther 2006;23:691-712. 